\title{
B-hadrons spectroscopy and decay properties with the ATLAS experiment
}

\section{Tomáš Jakoubek}

(Institute of Physics ASCR, Prague)

on behalf of the ATLAS Collaboration

tomas.jakoubek@cern.ch

QCD 2014, Montpellier, France

$$
\text { 01. } 07.2014
$$




\section{Outline}

- The ATLAS detector overview

- $B$-physics triggers

- Selection of related topics discussed today:

- Measurement of the $\Lambda_{b}{ }^{0}$ lifetime and mass [Phys. Rev. D 87, 032002 (2013)]

- Measurement of the parity-violating asymmetry parameter $\alpha_{b}$ and the helicity amplitudes for the decay $\Lambda_{b}{ }^{0} \rightarrow J / \psi \Lambda^{0}$ with the ATLAS detector [Phys. Rev. D 89092009 (2014)]

- Observation of an excited $B_{C}{ }^{ \pm}$meson state with the ATLAS detector [ATLAS-BPHY-2012-04-002, to be submitted to Phys. Rev. Lett.] 


\section{The ATLAS Detector}

- Multipurpose detector at LHC, optimized for high $p_{\mathrm{T}}$ physics and instantaneous luminosity up to $10^{34} \mathrm{~cm}^{-2} \mathrm{~s}^{-1}$

- Inner Detector

- $|\eta|<2.5$

- Solenoid $B=2 \mathrm{~T}$

- Si pixel, Si strips, Transition Radiation Tracker

- Impact parameter res. $15 \mu \mathrm{m}$ in $r \varphi, 100 \mu \mathrm{m}$ in $z$

- Momentum res. $\Delta p_{T} / p_{\mathrm{T}} \sim 0.04 \%$ $p_{\mathrm{T}}[\mathrm{GeV}] \otimes 1.5 \%$

- Muon Spectrometer

$$
\text { - }|\eta|<2.7
$$

Toroid average $B=0.5 \mathrm{~T}$ (barrel) or $1 \mathrm{~T}$ (end-caps)

Momentum res. $\Delta p_{\mathrm{T}} / p_{\mathrm{T}}<10 \%$

up to $\sim 1 \mathrm{TeV}$
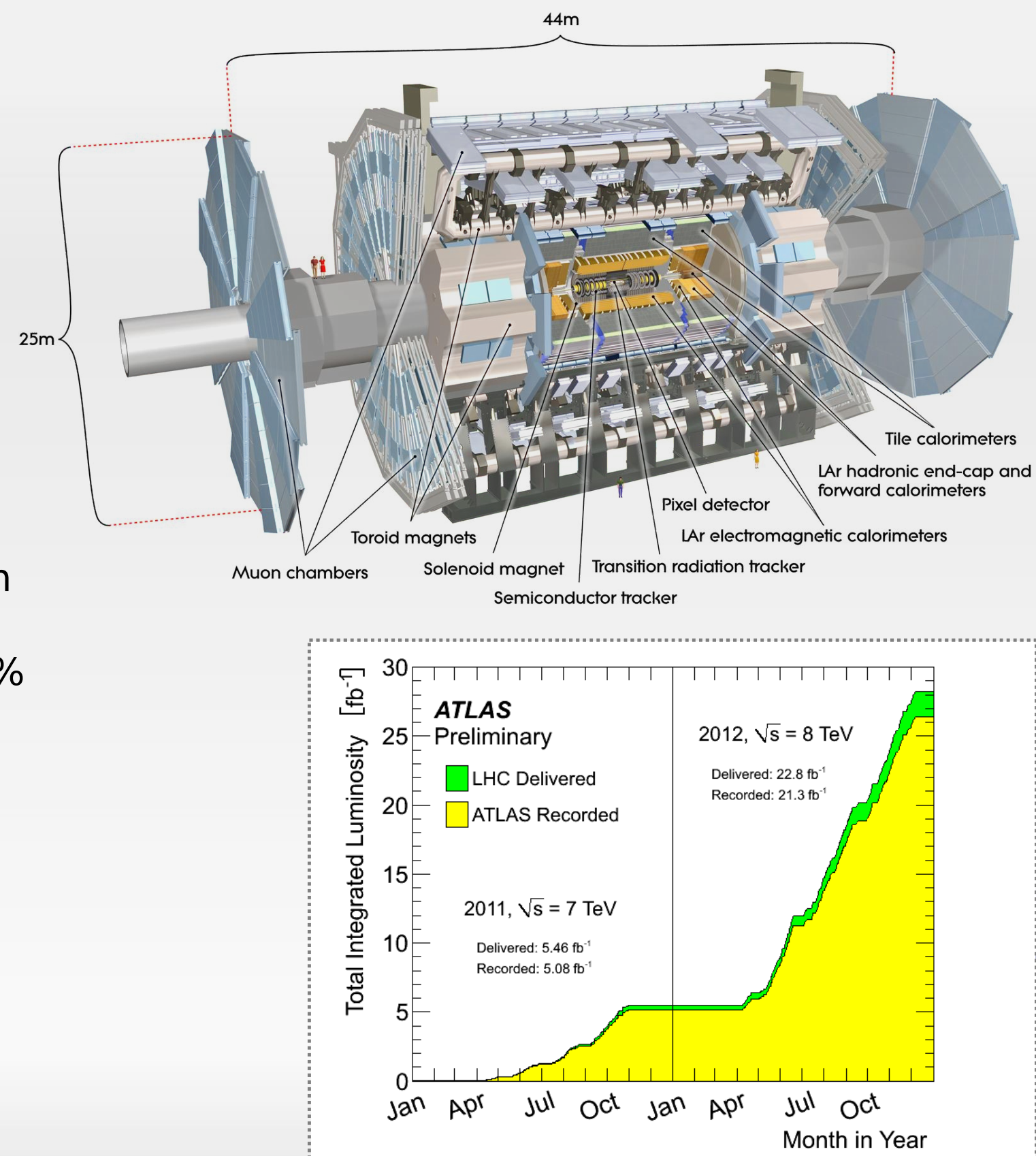


\section{B-physics triggers}

- ATLAS has 3-level trigger system (L1 - hardware, L2 and Event Filter computer farms)

- From $40 \mathrm{MHz}$ to $O(200) \mathrm{Hz}$

- $B$-physics triggers are based on single and di-muons...

- Topological triggers process two L1 muon Rols and refine results in the HLT with a good vertex fit and mass cut

- TrigDiMuon triggers require one L1 muon Rol and then search for a second muon in ID tracks

\begin{tabular}{|lll|}
\hline Trigger name & Mass window & \# of evts. \\
\hline 2mu4_Jpsimumu & 2.5-4.3 GeV & $14 \mathrm{M}$ \\
\hline 2mu4_Upsimumu & $8-12 \mathrm{GeV}$ & $9.1 \mathrm{M}$ \\
\hline 2mu4_Bmumu & $4-8.3 \mathrm{GeV}$ & $3.7 \mathrm{M}$ \\
\hline \multicolumn{3}{|c}{} \\
\hline \multicolumn{3}{|c|}{ Example with $2.3 \mathrm{fb}^{-1}$ data } \\
\hline
\end{tabular}

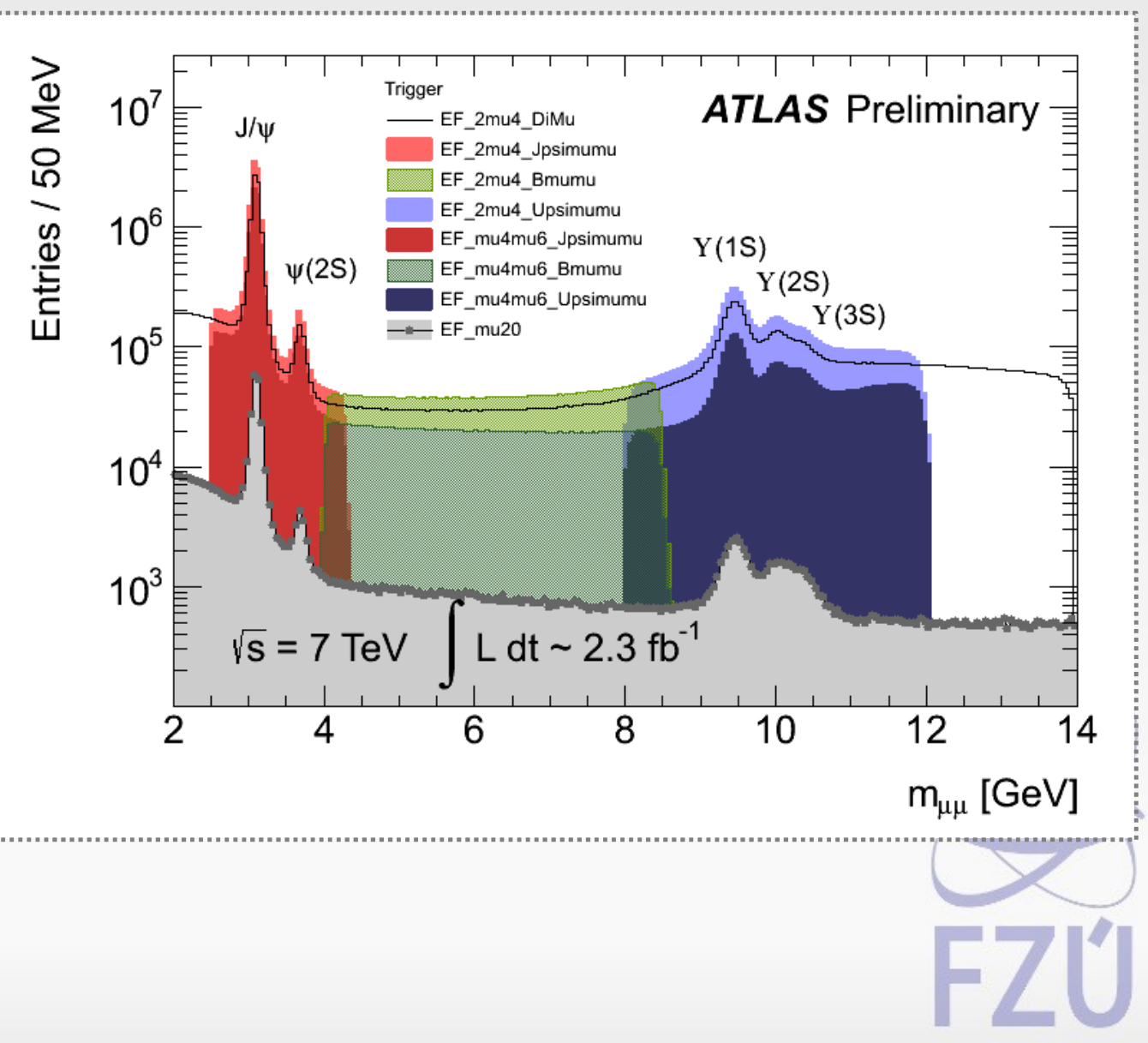




\section{Measurement of the $\Lambda_{b}{ }^{0}$ lifetime and mass}

- $\Lambda_{b}{ }^{0}$ is the lightest baryon containing a $b$-quark (5620 MeV)

- Not produced at $B$-factories $\Rightarrow$ currently hadron colliders are the only facilities to study $b$-baryons properties

- Lifetime, although measured by many experiments, still suffers from a large experimental uncertainty

- Data from 2011 proton-proton 7 TeV collisions, used integrated luminosity $4.9 \mathrm{fb}^{-1}$

- Reconstructed via $\Lambda_{b}{ }^{0} \rightarrow J / \psi\left(\mu^{-} \mu^{+}\right) \Lambda^{0}\left(p \pi^{-}\right)$

- Topological $J / \psi$ trigger chain

- Candidate selection:

- Global $\chi^{2} /$ n.d.o.f $<3$

- $p_{\mathrm{T}}\left(\Lambda^{0}\right)>3.5 \mathrm{GeV}$

- $L_{x y}\left(\Lambda^{0}\right)>10 \mathrm{~mm}$

$5.38 \mathrm{GeV}<m\left(J / \psi \Lambda^{0}\right)<5.90 \mathrm{GeV}$

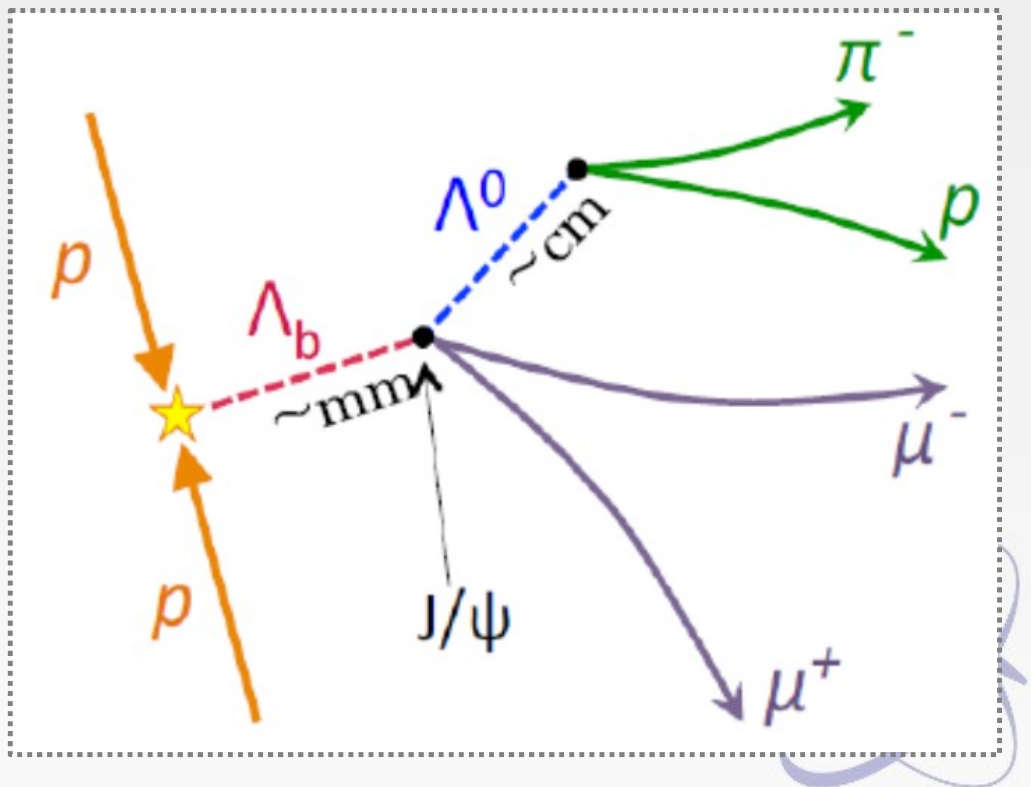




\section{Measurement of the $\Lambda_{b}{ }^{0}$ lifetime and mass}

- Unbinned maximum likelihood fit to mass and lifetime:

- Mass: signal - Gaussian, background - $1^{\text {st }}$ order polynomial

- Lifetime: signal - exponential function, prompt background - sum of $\delta$-function and a symmetric exponential distribution, nonprompt background - exponential function

- $m=5619.7 \pm 0.7$ (stat) \pm 1.1 (syst) $\mathrm{MeV}$

$\tau=1.449 \pm 0.036$ (stat) \pm 0.017 (syst) ps
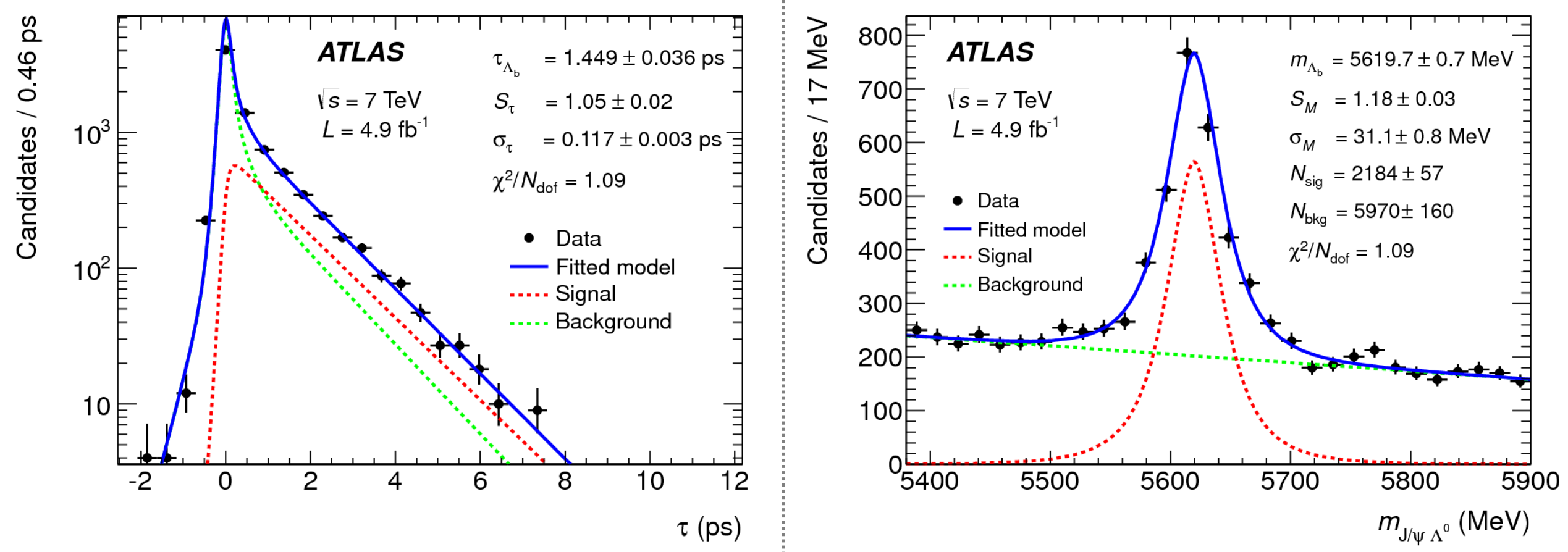


\section{Measurement of the $\Lambda_{b}{ }^{0}$ lifetime and mass}

- Comparison with other experiments
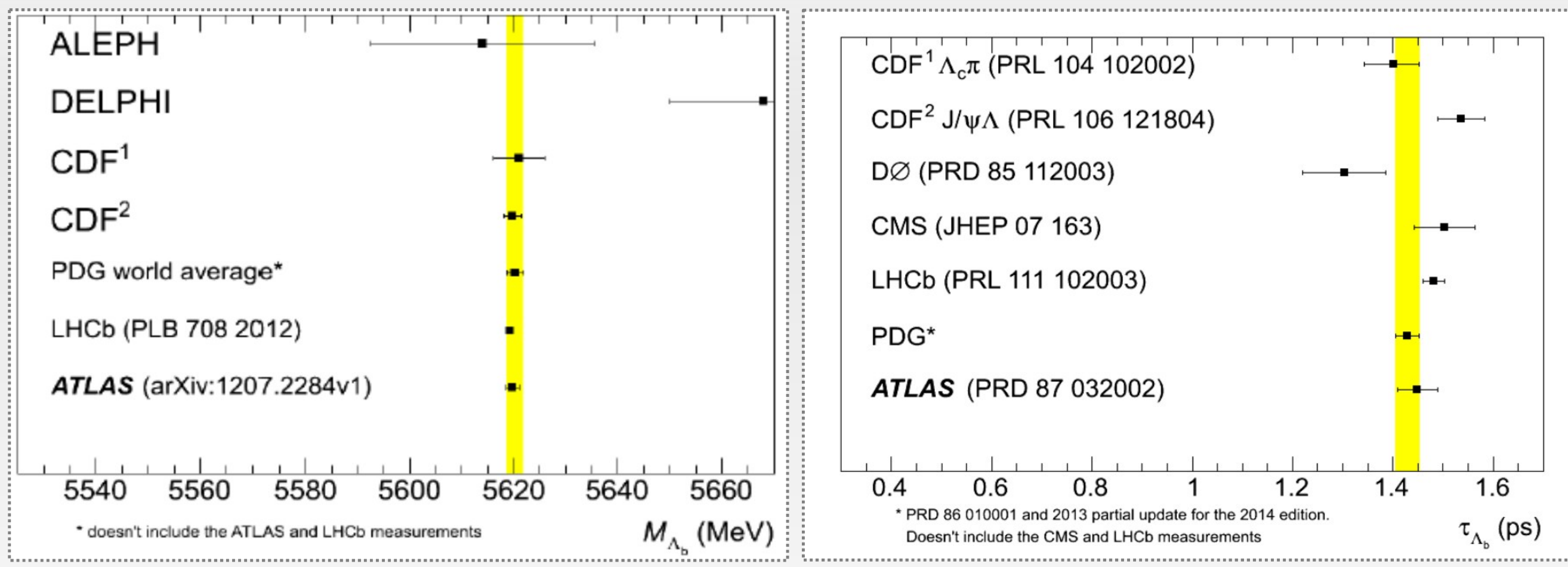


\section{Parity violation in the decay $\Lambda_{b}{ }^{0} \rightarrow J / \Psi \Lambda^{0}$}

- Parity violation - well-known feature of weak interactions - not maximal in decays of hadrons due to the presence of strongly bound spectator quarks

- Results of parity violation measurement can be used to test predictions made by different quark interaction models...

- $\mathbf{P Q C D}$ predicts $\alpha_{b}$ (parity-violating asymmetry parameter) to be in the range from $\mathbf{- 0 . 1 7}$ to $\mathbf{- 0 . 1 4}$, while calculations based on HQET predicts a value of $\sim \mathbf{0 . 7 8}$

- Decay described by 4 helicity amplitudes $A\left(\lambda_{\Lambda}, \lambda_{J / \psi}\right)$ normalized to 1 :

- $a_{+}=A(1 / 2,0)$

- $a_{-}=A(-1 / 2,0)$

- $b_{+}=A(-1 / 2,-1)$

- $b_{-}=A(1 / 2,1)$

- Parameter $\alpha_{b}=\left|a_{+}\right|^{2}-\left|a_{-}\right|^{2}+\left|b_{+}\right|^{2}-\left|b_{-}\right|^{2}$ 


\section{Parity violation in the decay $\Lambda_{b}{ }^{0} \rightarrow J / \Psi \Lambda^{0}$}

- Full angular PDF:

$w(\Omega, \vec{A}, P)=\frac{1}{(4 \pi)^{3}} \sum_{i=0}^{19} f_{1 i}(\vec{A}) f_{2 i}\left(P, \alpha_{\Lambda}\right) F_{i}(\Omega)$

$f_{1 i} \ldots$ bilinear combinations of the helicity amplitudes

$f_{2 i} \ldots=P \alpha_{\wedge}, P, \alpha_{\wedge}$ or 1

$F_{i} \ldots$ moments of the angular distribution

- $p p$ collisions symmetry + ATLAS $\eta$ symmetry

$\rightarrow$ overall polarization $P=0 \rightarrow$ reducing PDF from 20 to 6 terms

- Helicity amplitudes and decay asymmetry parameter for $\Lambda_{b}{ }^{0}$ extracted by least square fit from measured averages of each $F_{i}$ :

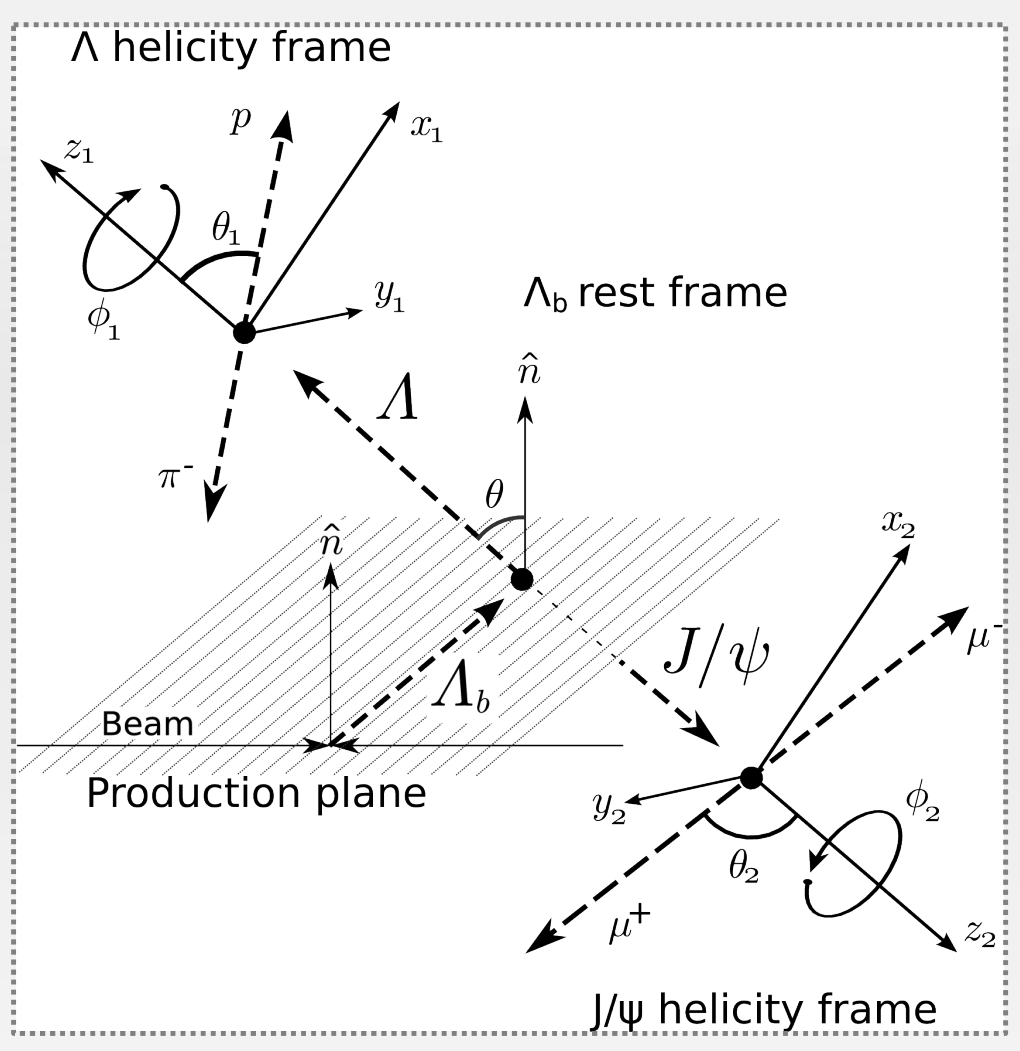

$$
\begin{gathered}
\left\langle F_{i}\right\rangle=\frac{1}{N^{\text {data }}} \sum_{n=1}^{N^{\text {data }}} F_{i}\left(\Omega_{n}\right) \\
\chi^{2}=\sum_{i} \sum_{j}\left(\left\langle F_{i}\right\rangle^{\text {expected }}-\left\langle F_{i}\right\rangle\right) V_{i j}^{-1}\left(\left\langle F_{j}\right\rangle^{\text {expected }}-\left\langle F_{j}\right\rangle\right)
\end{gathered}
$$

$V_{i j} \ldots$ covariance matrix of measured $\left\langle F_{i}\right\rangle_{i}\left\langle F_{i}\right\rangle^{\text {expected }}$ is evaluated from models including detector effects and depends on the helicity amplitudes 


\section{Parity violation in the decay $\Lambda_{b}{ }^{0} \rightarrow J / \Psi \Lambda^{0}$}

- Background contribution in the signal region can be estimated as an average of the values in the two sidebands
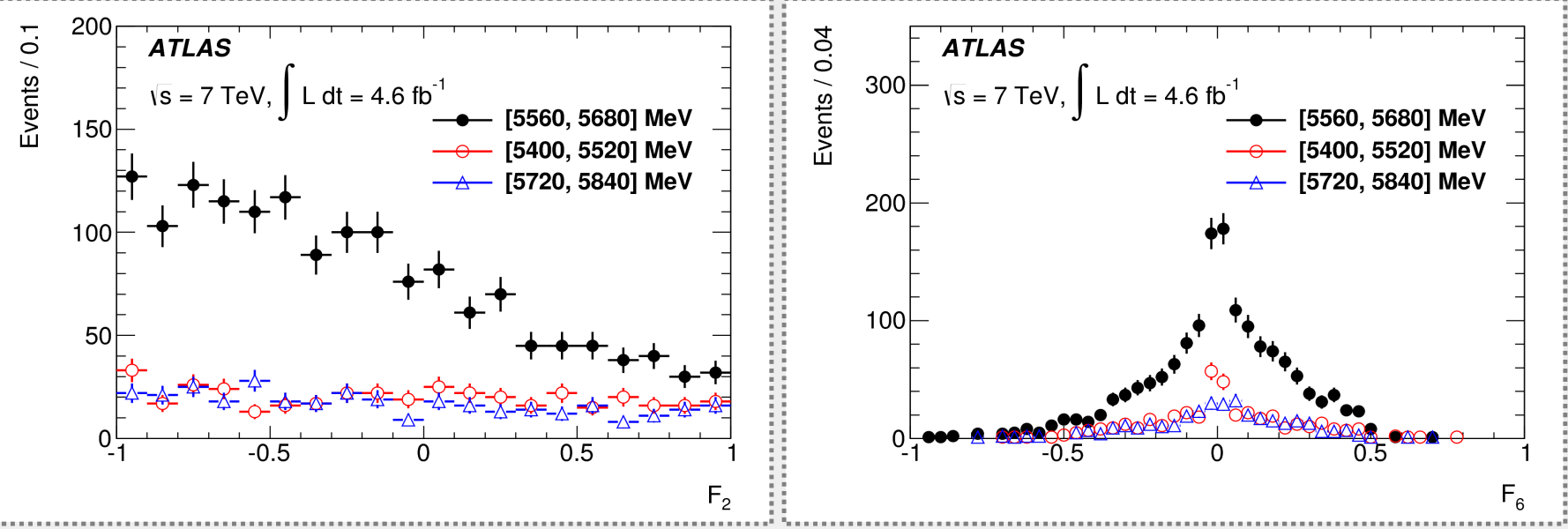

- Results checked by comparing $F_{i}$ distributions for data with weighted signal MC plus sideband background
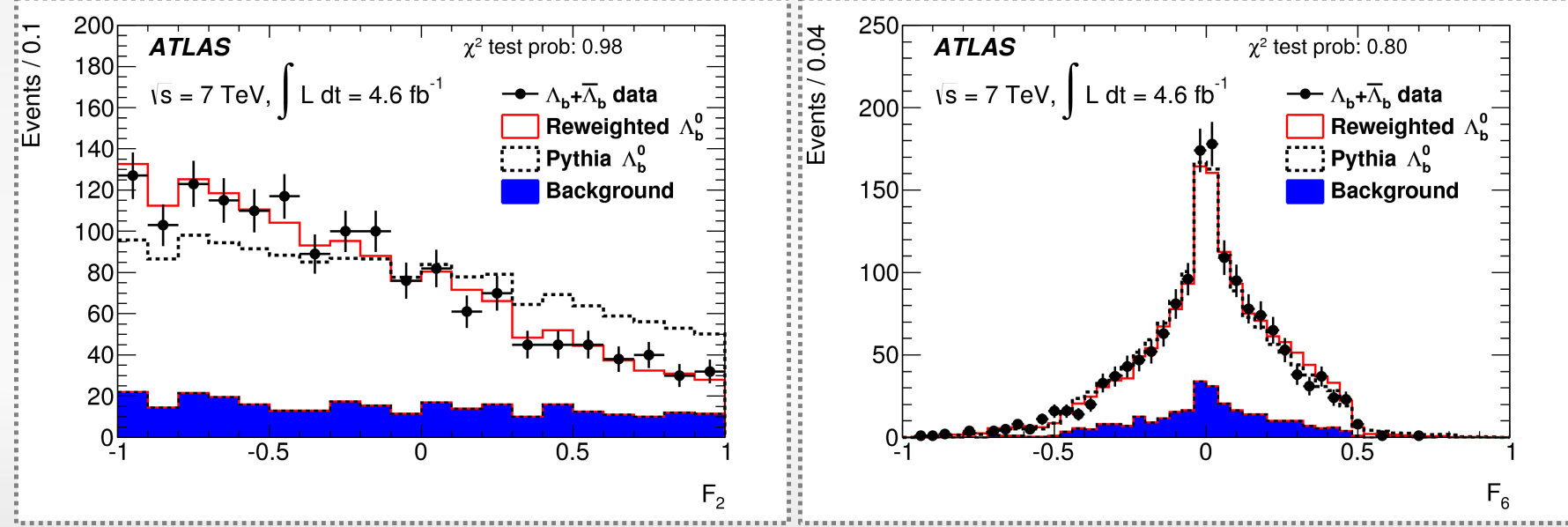


\section{Parity violation in the decay $\Lambda_{b}{ }^{0} \rightarrow J / \psi \Lambda^{0}$}

- Helicity amplitudes:

$$
\begin{aligned}
& \left|a_{+}\right|=0.17_{-0.17}^{+0.12} \text { (stat) } \pm 0.09 \text { (syst) } \\
& \left|a_{-}\right|=0.59_{-0.07}^{+0.06} \text { (stat) } \pm 0.03 \text { (syst) } \\
& \left|b_{+}\right|=0.79_{-0.05}^{+0.04} \text { (stat) } \pm 0.02 \text { (syst) } \\
& \left|b_{-}\right|=0.08_{-0.08}^{+0.13} \text { (stat) } \pm 0.06 \text { (syst) }
\end{aligned}
$$

- Decay asymmetry parameter:

$$
\alpha_{b}=0.30 \pm 0.16 \text { (stat) } \pm 0.06 \text { (syst) }
$$

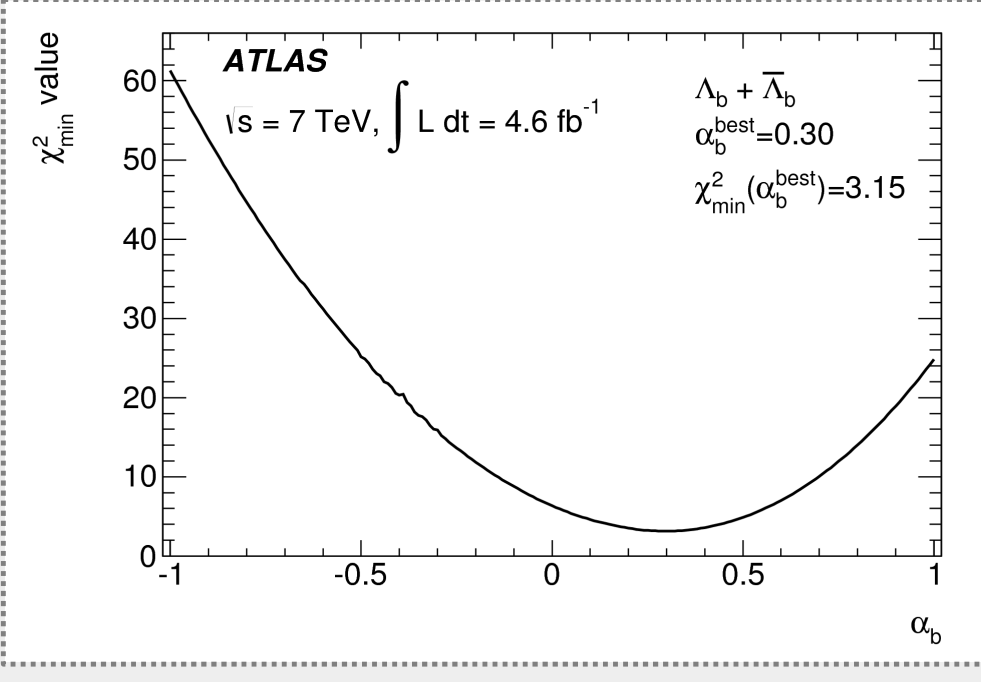

- Large $\left|a_{-}\right|$and $\left|b_{+}\right| \rightarrow$ negative helicity states for $\Lambda^{0}$ preferred

- $\Lambda^{0}$ and $J / \psi$ are highly polarized in the direction of their momenta

- $\alpha_{b}$ consistent with LHCb (Phys. Lett. B 724 (2013) 27):

$$
\alpha_{b}=0.05 \pm 0.17 \text { (stat) } \pm 0.07 \text { (syst) }
$$

- $\alpha_{b}$ differs from pQCD expectation (-0.17 to -0.14$)$ by $\sim 2.6 \sigma$ and from HQET expectation ( 0.78 ) by $\sim 2.8 \sigma$ Analysis using 2012 data is ongoing 


\section{Observation of an excited $B_{c}{ }^{ \pm}$state}

- Excited states of $B_{C}{ }^{ \pm}$meson have not previously been observed

- Spectrum and properties predicted by nonrelativistic potential models, pQCD, and lattice calculations

- Predicted $B_{C}{ }^{ \pm}(2 S)$ mass is in the range 6835 - $6917 \mathrm{MeV}$, production cross-section should be $15-20 \%$ of the ground state;

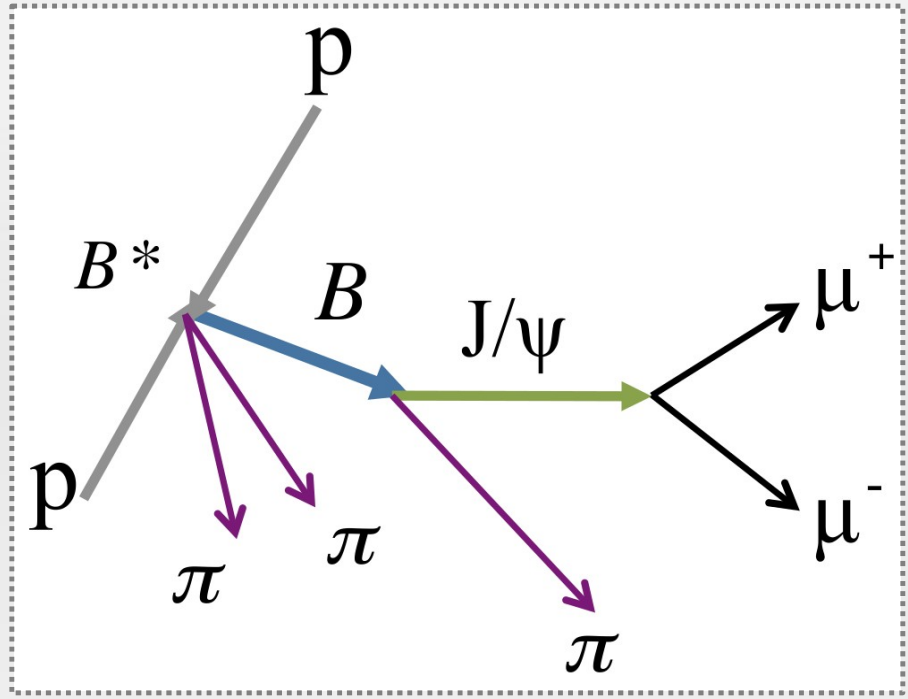
production of $P$ and $D$ states is highly suppressed relative to that of $B_{C}{ }^{ \pm}(2 S)$ state

- Data from 2011 (7 TeV) and 2012 (8 TeV) proton-proton collisions, used integrated luminosity $4.9 \mathrm{fb}^{-1}(2011)+19.2 \mathrm{fb}^{-1}$ (2012)

- New state observed through its hadronic transition to the ground state, with $B_{c}^{ \pm} \rightarrow J / \psi\left(\mu^{-} \mu^{+}\right) \Pi^{ \pm}$

To minimize uncertainties due to detector resolution, peaks are sought in the distribution of the variable

$$
\mathrm{Q}=m\left(B_{C}^{ \pm} \pi \pi\right)-m\left(B_{C}^{ \pm}\right)-2 m\left(\pi^{ \pm}\right)
$$




\section{Observation of an excited $B_{c}$ meson state}

- Invariant mass of $B_{C}{ }^{ \pm} \rightarrow J / \psi \pi^{ \pm}$(unbinned maximum likelihood fit)
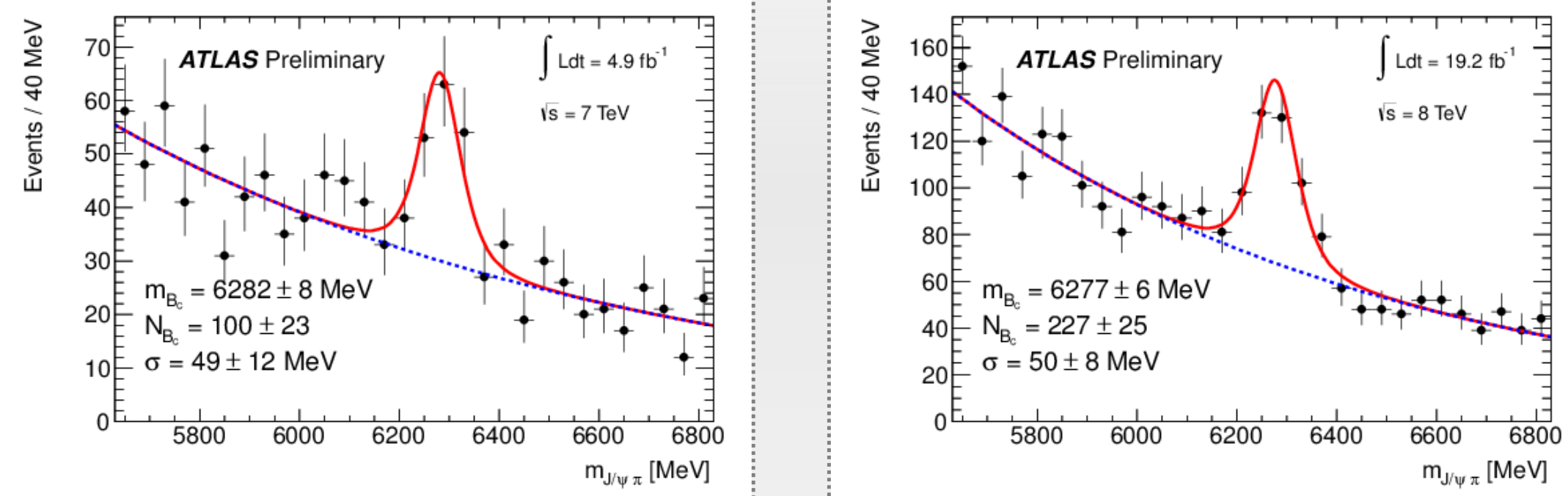

- The $m\left(B_{c}{ }^{ \pm} \pi \pi\right)-m\left(B_{c}{ }^{ \pm}\right)-2 m\left(\pi^{ \pm}\right)$mass difference distribution, wrongcharge combinations also shown (unbinned maximum likelihood fit)
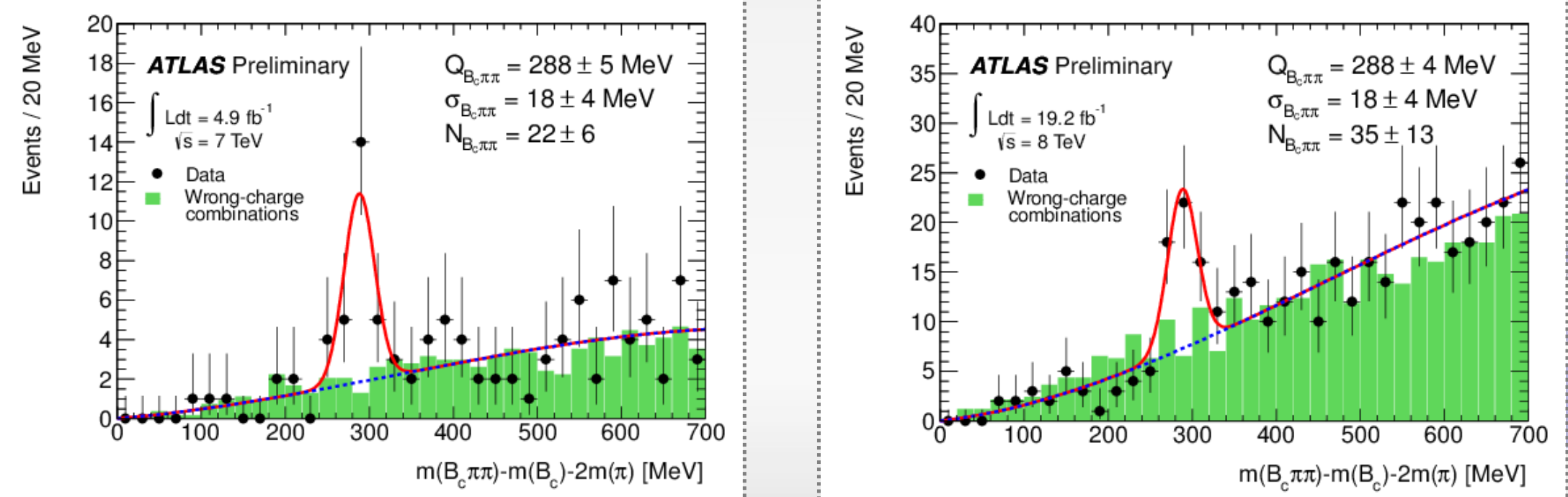


\section{Observation of an excited $B_{c}$ meson state}

- Systematics:

- Uncertainty coming from the statistical uncertainty of the ground state mass mostly cancels in $Q$-value distribution

- Uncertainty from the fit, estimated with various fit models including toy $\mathrm{MC}$ studies basing on the background shape from the wrong-charge combinations $(\sim 3.4 \mathrm{MeV})$ and varying the fit mass range from $0-700 \mathrm{MeV}$ to $0-1500 \mathrm{MeV}(\sim 1.2 \mathrm{MeV})$; these propagate to $m\left(B_{C}{ }^{ \pm}(2 S)\right)$ as $\sim 3.6 \mathrm{MeV}$

- Mass of the peak

$$
m\left(B_{c}{ }^{ \pm}(2 S)\right)=6842 \pm 7 \text { (stat) } \pm 5 \text { (syst) MeV }
$$

consistent with the theory $(6835-6917 \mathrm{MeV})$, as well as the decay mode

- 5.2 sigma observation for the combination of 7 and $8 \mathrm{TeV}$ datasets 


\section{Summary and Outlook}

- ATLAS has produced impressive and competitive results in heavy quark hadrons spectroscopy

- Presented today:

- $\Lambda_{b}^{0}$ mass and lifetime measurement in agreement with other experiments

- Parity violation parameter in decay $\Lambda_{b}{ }^{0} \rightarrow J / \psi \Lambda^{0}$ consistent with $\mathrm{LHC} b$ result, but lies between two theoretical predictions (pQCD and HQET); updates with 2012 data ongoing

- Observation of a new excited $B_{C}{ }^{ \pm}$meson state, $B_{C}{ }^{ \pm}(2 S)$, mass consistent with the theory

- For RUN-2, insertion of Pixel Insertable B-Layer (IBL) should provide increasing vertexing performance

- More public results on ATLAS B-physics TWiki page:

https://twiki.cern.ch/twiki/bin/view/AtlasPublic/BPhysPublicResults 


\section{BACKUP}

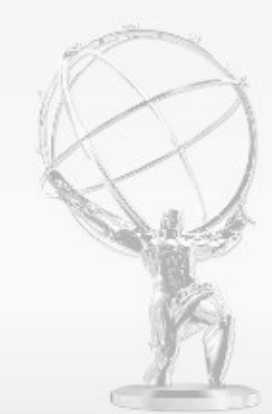




\section{Observation of an excited $B_{c}$ meson state}

- Topological $J / \psi$ trigger chain

- Early 2011: both muons with $p_{\mathrm{T}}>4 \mathrm{GeV}$

- Later 2011 and 2012: $p_{\mathrm{T}}\left(\mu_{\mathrm{H}}\right)>6 \mathrm{GeV}, p_{\mathrm{T}}\left(\mu_{\mathrm{S}}\right)>4 \mathrm{GeV}$

- $B_{C}{ }^{ \pm}$candidate selection:

- $J / \psi$ muons $p_{\mathrm{T}}$ cuts according to trigger

- $\pi$ track $p_{\mathrm{T}}>4 \mathrm{GeV}$ in both 2011 and 2012

- $p_{T}\left(B_{C}{ }^{ \pm}\right)>15 \mathrm{GeV}(2011)$ or $>18 \mathrm{GeV}$ (2012)

- Cut on $\pi$ track impact parameter ( $X Y$-plane projection) to reduce combinatorial background: $d_{x y}{ }^{0} / \sigma\left(d_{x y}{ }^{0}\right)>5(2011)$ or $>4.5$ (2012)

- $J / \psi \pi$ vertex $\chi^{2} /$ n.d.o.f $<2$ (2011) or $<1.5$ (2012)

- Excited $B_{C}{ }^{ \pm}$candidate selection:

- $B_{C}{ }^{ \pm}$candidate mass in $3 \sigma$ interval around its PDG mass

- $\pi$ tracks with $p_{\mathrm{T}}>400 \mathrm{MeV}$; from the corresponding primary vertex (PV)

- Refitted triplet of $B_{C}{ }^{ \pm}$tracks and the pair of $\pi$ tracks from PV must intersect in two separate $B_{C}{ }^{ \pm}$and $B_{C}{ }^{ \pm}(2 S)$ vertices

Taking a candidate with the best $\chi^{2}$ of the combined cascade fit with the ground state 